\title{
Broken promises and quantum algorithms
}

\author{
Adam Brazier and Martin B. Plenio \\ QOLS, Blackett Laboratory, \\ Imperial College, \\ Prince Consort Road, London, SW7 2BW, UK
}

November 22, 2018

\begin{abstract}
In the black-box model, promise problems are the only ones that admit a quantum exponential speedup over the best classical algorithm in terms of query complexity. The most prominent example of this is the Deutsch-Jozsa algorithm. More recently, Wim van Dam put forward an algorithm for unstructured problems (ie, those without a promise). We consider the Deutsch-Jozsa algorithm with a less restrictive (or 'broken') promise and study the transition to an unstructured problem. We compare this to the success of van Dam's algorithm. These are both compared with a standard classical sampling algorithm. The Deutsch-Jozsa algorithm is surprisingly good as the problem becomes less structured and is always better than the van Dam algorithm for low numbers of queries.
\end{abstract}

\section{Introduction}

It is known that for a quantum algorithm to achieve a black-box exponential speedup over a classical one, the problem in question must be a 'promise problem' (Buhrman et al., [1]). In the Black Box model of computation, where the oracle contains a set of $N$ Boolean variables $X=$ $\left(x_{0}, x_{1}, x_{2} \ldots x_{N-1}\right)$, about the properties of which questions can be asked (eg, 'do all the $x_{i}$ have the value 1', or 'do the $x_{i}$ contain at least one 1'), a partial or promise problem is one with a restriction on the $X \mathrm{~s}$ that are allowed.

The Deutsch-Jozsa problem is an example of such a problem, where the question is one of deciding whether or not a given function is 'balanced' or 'constant' (each of these criteria describes a set of possible $X$ from the set of all possible $N$-bit strings and so comprises a promise as described above). We consider a relaxation of the promise on this problem (ie, we allow extra possible $X$ ) and consider how this affects the efficacy of the Deutsch-Jozsa algorithm as the weakening of the promise is increased and the problem becomes less structured. 
Following an introduction to the standard Deutsch-Jozsa problem (in section 2), we modify/weaken the promise on the Deutsch-Jozsa problem in section 3 and then consider the performance of the Deutsch-Jozsa algorithm on this modified problem. A classical algorithm based on sampling is devised to address the new problem and its success probability is considered. In section 3.3] we introduce van Dam's Quantum Oracle Interrogation algorithm [3] (which is designed for unstructured problems) and consider how well it can be used to solve the modified problem and compare its performance with that of the Deutsch-Jozsa algorithm as the problem becomes less structured; this allows us to determine in which regimes one might prefer to use which algorithm.

\section{The Deutsch-Jozsa problem and algorithm}

The Deutsch-Josza problem is to recognise whether a Boolean function $\mathrm{f}$, $\{0,1\}^{n} \stackrel{f}{\rightarrow}\{0,1\}$ is 'balanced' or 'constant' (that the function has one of these two properties is the promise on the problem). A constant function is one for which $f(x)$ evaluates the same, for all $x \in\{0,1\}^{n}$ (ie, either all 1 s or all $0 \mathrm{~s})$. A balanced function is one in which $f(x)$ evaluates to 0 for exactly half of the $x$ and 1 for the other half. The Deutsch-Jozsa algorithm [4- 6 ] solves this problem with one query and with no probability of error.

The input state for the Deutsch-Josza algorithm is an equal superposition of all the $|x\rangle$ such that $x \in\{0,1\}^{n}$ (constructed by acting on $|00 \ldots 0\rangle$ with $\left.H^{\otimes n}\right)$, with an ancilla qubit in the state $\frac{1}{\sqrt{2}}(|0\rangle-|1\rangle)$. This is then acted on with the operator $U_{f}: U_{f}|x\rangle|y\rangle \rightarrow|x\rangle|y \oplus f(x)\rangle$ which, given that $|y\rangle$ is the ancilla bit already described and ' $\oplus$ ' represents addition modulo 2 , has the effect of introducing a phase $(-1)^{f(x)}$ to the state $|x\rangle$. Finally an $n$-bit Hadamard is applies to the first $n$ bits (the ancilla is discarded) and then the resulting state is measured along $|z=0\rangle$. The process, omitting the ancilla quibit, is summarised below:

$$
\begin{aligned}
|00 \ldots 0\rangle & \stackrel{H^{\otimes n}}{\rightarrow} \frac{1}{\sqrt{2^{n}}} \sum_{x=0}^{x=2^{n}-1}|x\rangle \\
& \stackrel{U_{f}}{\rightarrow} \frac{1}{\sqrt{2^{n}}} \sum_{x=0}^{x=2^{n}-1}(-1)^{f(x)}|x\rangle \\
& \stackrel{H^{\otimes n}}{\rightarrow} \frac{1}{2^{n}} \sum_{x, z=0}^{x, z=2^{n}-1}(-1)^{f(x)+x . z}|z\rangle,
\end{aligned}
$$

where ' $x . z$ ' refers to the scalar product between bitstrings $\left(x . z=x_{1} z_{1} \oplus\right.$ $\left.x_{2} z_{2} \oplus \ldots \oplus x_{n} z_{n}\right)$. Consideration of the state $|z=0\rangle$ reveals that its amplitude is 1 if the function is constant and 0 if the function is balanced, so 
measuring along $|z=0\rangle$ will allow perfect differentiation between the cases of the function being balanced or constant. Achieving this feat classically would require examination of half of the values of $f(x)$ plus one, for a total of $\frac{2^{n}}{2}+1$ queries and, therefore, an exponential disadvantage as compared to the quantum algorithm.

If error is allowed, a classical algorithm needs far less queries to achieve a small error (see, eg, 2]). This algorithm progresses by looking at bits and as soon as a bit is observed which is different to previous bits, the algorithm halts and returns the result 'balanced', else it terminates after $k$ bits examined (ie, $k$ queries to the algorithm) and returns 'constant'. The only possibility of error, therefore, is when $k$ identical bits are observed (in which case the result 'constant' is returned) and the function is, in fact, balanced; the probability of this occurring is just the chance of selecting $k$ identical objects from a sample of two species of equal number.

$$
P_{\text {fail }}=2 p \frac{\frac{N}{2} C_{k}}{{ }^{N} C_{k}}
$$

where $p$ is the probability that the function is actually balanced and we use

the notation ${ }^{a} C_{b}$ to indicate $\left(\begin{array}{c}a \\ b\end{array}\right)=\frac{a !}{b !(a-b) !}$. If the constant and balanced cases are equally likely and $k \ll N$,

$$
P_{\text {fail }} \simeq \frac{1}{2^{k}}
$$

and we can see that a small number of queries $k$ can produce an accurate answer.

\section{Breaking the Promise}

We can imagine weakening the promise on the problem in the following way: introducing $y$ bit-flips into the $N=2^{n}$ bit string at random positions that characterises the function $f$ would correspond to saying that the function is 'nearly always balanced' or 'nearly always constant', up to $y$ deviations from the promise. In this section, when we say 'constant' we are actually referring to 'nearly constant', and similarly in the case of 'balanced'.

\subsection{Deutsch-Jozsa algorithm with broken promise}

The Deutsch-Jozsa (DJ) algorithm can be used unmodified on the new problem. If the function $f(x)$ is actually balanced, then when in the coefficient of $|z=0\rangle$ in equation (11), we see that the cancellation caused by equal numbers of terms with $f(x)=1$ and $f(x)=0$ in the index of -1 is no longer 
achieved. We now have $\left(\frac{N}{2}+y\right)$ of \pm 1 in the phase of $|z=0\rangle$ and $\left(\frac{N}{2}-y\right)$ of $\mp 1$. There is, therefore, a non-zero coefficient of $|z=0\rangle, \alpha_{0}$ :

$$
\alpha_{0}= \pm \frac{2 y}{2^{n}}
$$

which leads to an error probability $P_{\text {bal }}$ of (given that error in this case is measuring $|z=0\rangle)$ :

$$
P_{\text {bal }}=y^{2} 2^{2-2 n} .
$$

For $y$ weakenings in the promise on the actually constant $n$-bit function $f(x)$ we find that for the D-J algorithm we have:

$$
\alpha_{0}= \pm\left(1-\frac{2 y}{2^{n}}\right)
$$

It is an error, in the case where $f(x)$ is constant, if we do do not observe $|z=0\rangle$, so our error probability in this case $P_{\text {con }}$ is given by:

$$
P_{\text {con }}=y 2^{2-n}-y^{2} 2^{2-2 n},
$$

and we see that the error is worse if the function is actually constant than if it is balanced ${ }^{1}$. Assuming that the probability that the function $f(x)$ is balanced is $p$, the error probability $P_{\text {fail }}$ from one query to the quantum algorithm is:

$$
\begin{aligned}
P_{\text {fail }} & =p P_{\text {bal }}+(1-p) P_{\text {con }} \\
& =p y 2^{2-n}-p y^{2} 2^{2-2 n}+(1-p) y^{2} 2^{2-2 n} \\
& =p y 2^{2-n}+(1-2 p) y^{2} 2^{2-2 n},
\end{aligned}
$$

which, in the case where each case is equally probable $\left(p=\frac{1}{2}\right)$, is given by:

$$
P_{\text {fail }}=y 2^{1-n}
$$

for a single query.

The chance of the quantum algorithm failing we assess over $k$ queries by majority decision (for direct comparison with van Dam's algorithm and the classical case); this is the chance that $\geq \frac{k}{2}$ of the results return the wrong result (all evaluated on copies of the same function).

$$
P_{\text {fail }}=\sum_{r=\frac{k}{2}}^{k}\left({ }^{k} C_{r}\left(p P_{\text {bal }}^{r}\left(1-P_{\text {bal }}\right)^{k-r}+(1-p) P_{\text {con }}^{r}\left(1-P_{\text {con }}\right)^{k-r}\right)\right),
$$

\footnotetext{
${ }^{1}$ This is because any redistribution of the outcome probabilities in the 'constant' case will give rise to error (because it gives rise to probability of observing $|z \neq 0\rangle$, whereas the only redistribution of the outcome probabilities that will cause error in the 'balanced' case is that which specifically causes an increase in the probability of observing $|z=0\rangle$
} 
Some examples of this can be seen in figures 1 and 2 for $p=\frac{1}{2}$, whereupon we see that the DJ algorithm is robust for lower values of $y$ (up to about $\left.y \simeq \frac{N}{8}\right)$.

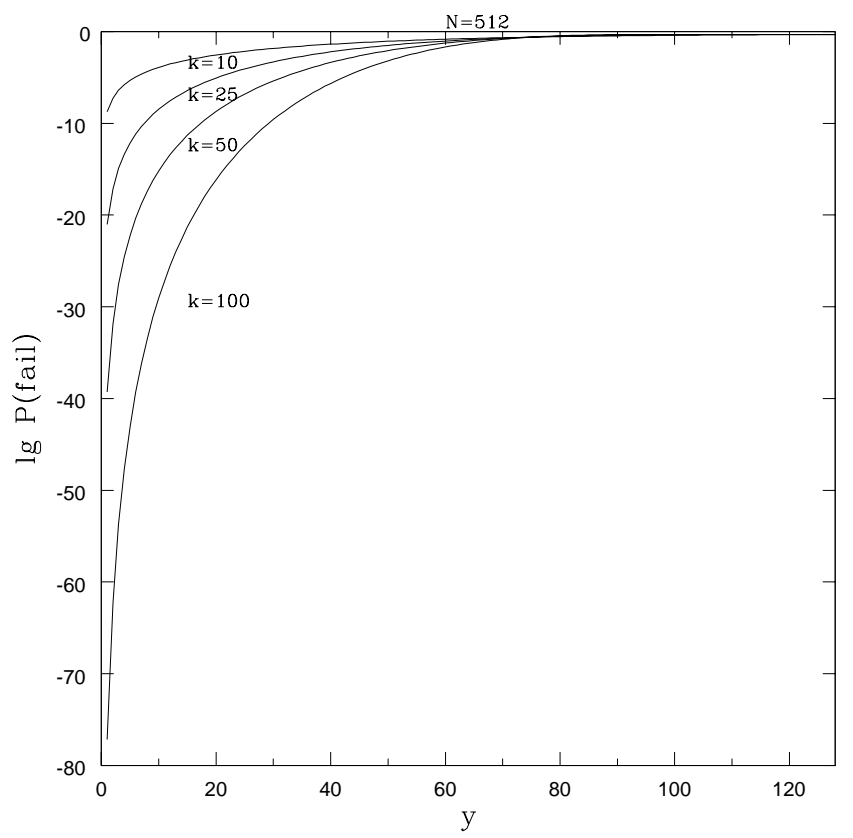

Figure 1: Failure probability for DJ algorithm on broken promise against number of weakenings $y$

\subsection{Classical algorithm with broken promise}

Once we allow weakenings of the promise, the classical algorithm from section 2 becomes particularly poor; whereas previously the algorithm only failed in the case that the algorithm was actually balanced, as the number of weakenings increases it becomes more probable that a constant function will be described as balanced and this probability dominates the error probability relatively quickly. The existing algorithm is closely tied to the promise being unbroken, but a new algorithm, based on sampling, is easily designed.

This algorithm queries $k$ values of the function and then makes a decision depending on the relative numbers of bits in the sample, by counting the number of $0 \mathrm{~s}$ in the sample, $k_{0}$. The proportion of $0 \mathrm{~s}$ in the sample is used as an estimator, $\hat{p}_{0}^{c l}=\frac{k_{0}}{k}$ for the number of $0 \mathrm{~s}$ in the full $N$ bit string of $1 \mathrm{~s}$ and 0s that describes $f$. The algorithm then infers balanced if $0.25<\hat{p}_{0}^{c l}<0.75$ or constant otherwise. This estimator is unbiased since its expected value $E\left(\hat{p}_{0}^{c l}\right)=N_{0} / N$, the true proportion of zeros in the string, independent of the degree of weakening. This algorithm avoids the severe failure in the case 


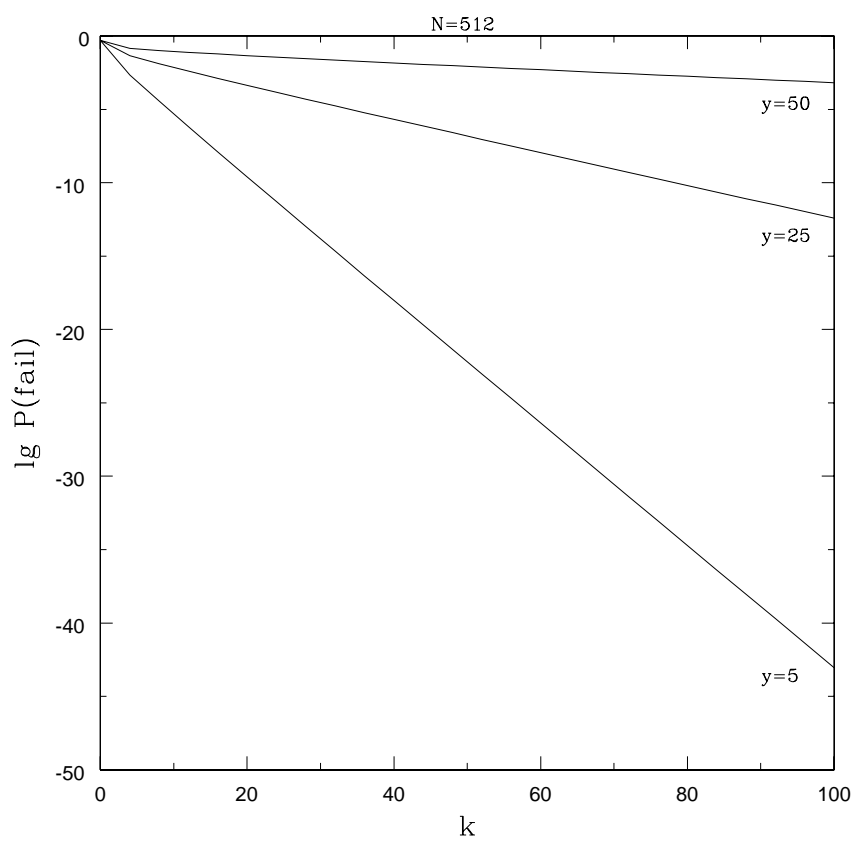

Figure 2: Failure probability for DJ algorithm on broken promise against number of queries $k$

of 'actually constant' that the previous classical algorithm from section 2 suffers from.

This new classical algorithm fails if we have

- 'constant' (ie, 'weakened constant') string and yet observe the number of $0 \mathrm{~s}, k_{0}$ in the $k$ queries such that $\frac{k}{4} \leq k_{0} \leq \frac{3 k}{4}$

- 'balanced' and yet observe a number of $0 \mathrm{~s}, k_{0}$, in the $k$ queries such that $k_{0} \leq \frac{k}{4}$ or $k_{0} \geq \frac{3 k}{4}$

so in both of these cases, the wrong result is returned.

If we describe the event 'observing $\frac{k}{4} \leq k_{0} \leq \frac{3 k}{4}$ ' as $B$ and the property 'string is balanced' as $A_{1}$ and the property 'string is constant' as $A_{2}$, then we can write an expression for the probability of algorithm failure, $P_{\text {fail }}$ in terms of the conditional probabilities of deciding on one case given that the other is actually the case:

$P_{\text {fail }}=p\left(1-\frac{1}{2} P\left(B \mid A_{1 a}\right)-\frac{1}{2} P\left(B \mid A_{1 b}\right)\right)+(1-p)\left(\frac{1}{2} P\left(B \mid A_{2 a}\right)+\frac{1}{2} P\left(B \mid A_{2 b}\right)\right)$, 
in which the subscript $a$ refers to the case when there is an excess of zeroes in the $N$ values of $f(x)$ and $b$ refers to when there is an excess of ones and, as before, $p$ is the probability that the function is actually balanced, so that:

$$
\begin{aligned}
& P\left(B \mid A_{1 a}\right)=\sum_{k_{0}=\frac{k}{4}}^{\frac{3 k}{4}} \frac{\left(\frac{N}{2}+y\right) C_{k_{0}} \cdot{ }^{\left(\frac{N}{2}-y\right)} C_{\left(k-k_{0}\right)}}{{ }^{N} C_{k}}, \\
& P\left(B \mid A_{1 b}\right)=\sum_{k_{0}=\frac{k}{4}}^{\frac{3 k}{4}} \frac{\left(\frac{N}{2}-y\right) C_{k_{0}} \cdot{ }^{\left(\frac{N}{2}+y\right)} C_{\left(k-k_{0}\right)}}{{ }^{N} C_{k}}, \\
& P\left(B \mid A_{2 a}\right)=\sum_{k_{0}=\frac{k}{4}}^{\frac{3 k}{4}} \frac{(N-y) C_{k_{0}} \cdot{ }^{y} C_{\left(k-k_{0}\right)}}{{ }^{N} C_{k}} \\
& P\left(B \mid A_{2 b}\right)=\sum_{k_{0}=\frac{k}{4}}^{\frac{3 k}{4}} \frac{{ }^{y} C_{k_{0}} \cdot{ }^{(N-y)} C_{\left(k-k_{0}\right)}}{{ }^{N} C_{k}} .
\end{aligned}
$$

The results can be seen in figures 3 and 4 for the case where $p=\frac{1}{2}$.

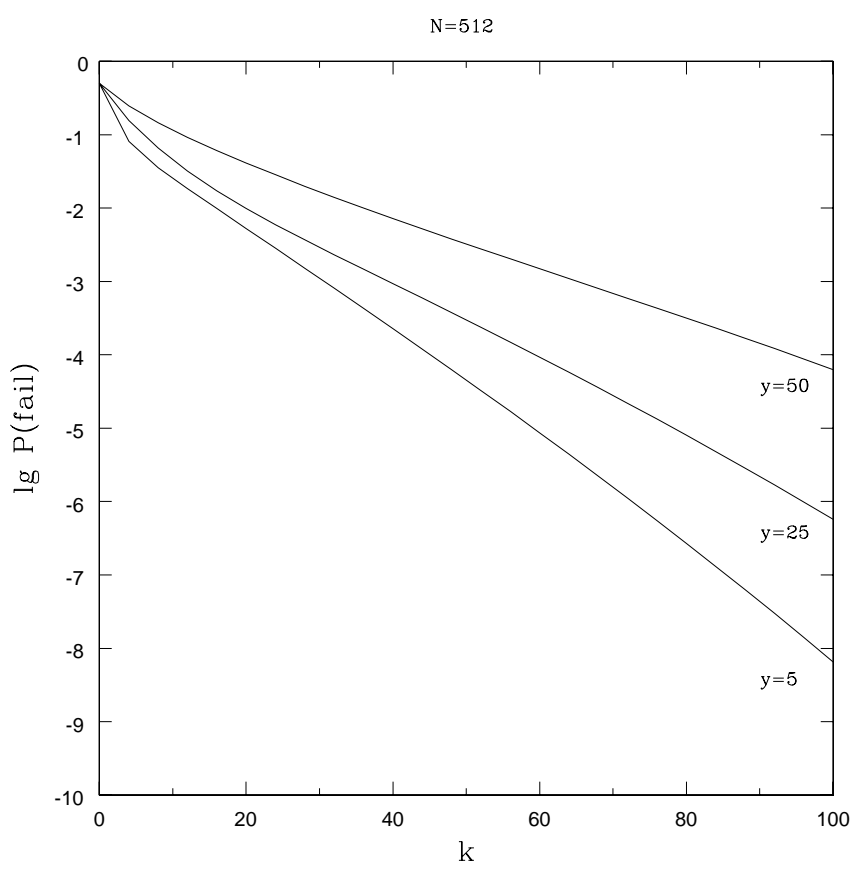

Figure 3: Failure probabilities for classical algorithm when promise is broken in terms of number of queries $k$ 


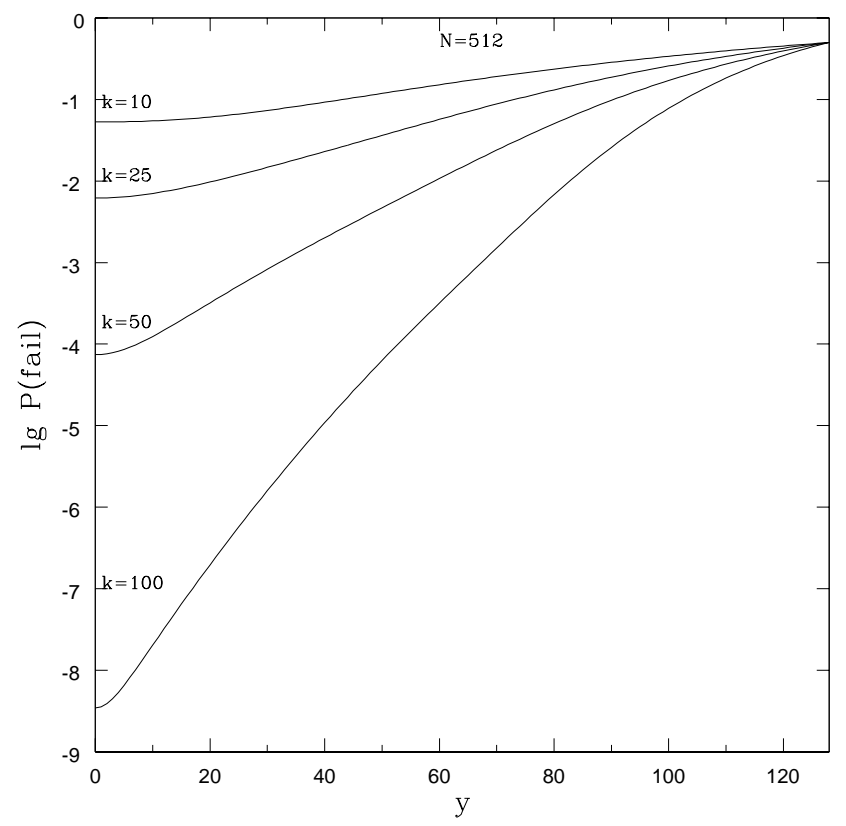

Figure 4: Failure probabilities for classical algorithm when the promise is broken in terms of weakenings in the promise, $y$

Given that much of the power of the quantum algorithm lies in its ability to establish the nature of $f(x)$ with a single query it is interesting to consider how many classical queries are required to achieve the same probability of success as one quantum query. With $k, y, k y \ll N$, it turns out that the number of queries $k$ to the classical algorithm to achieve the same success probability as one query to the Deutsch-Jozsa algorithm obeys (for $y, k \ll$ $N)$ :

$$
2\left(\frac{y}{N}\right)>\left[\sum_{k_{0}>\frac{3 k}{4}, k_{0}<\frac{k}{4}}{ }^{k} C_{k_{0}}\right]\left(\frac{1}{2}\right)^{k-1}
$$

and we see that the advantage of the quantum over the classical case is constant where $\frac{y}{N}$ is constant (which would correspond to a fixed chance of a given function value being 'flipped').

\subsection{Quantum Oracle Interrogation}

Since reducing the strength of the promise is akin to making the problem less structured we compare the power of the DJ algorithm with that of an algorithm that is specficially tailored for completely unstructured problems, 
the Quantum Oracle Interrogation algorithm of van Dam [3] [7] (WVD). In this case, there is no promise on the problem (ie, all possible $X$ are allowed).

An N-bit function $f$ can be be described by a string consisting of the values of $f(x)$; this is represented as a state $|F\rangle=\left|f_{0} f_{1} \ldots f_{N-1}\right\rangle$, where $f_{x}=f(x)$, an operator $A_{k}$ is introduced, the action of which depends on the Hamming weight $\|x\|$ of the state $|x\rangle$ (ie the number of $1 \mathrm{~s}$ in the binary representation of $x$ ):

$$
A_{k}|x\rangle|b\rangle=\left\{\begin{array}{ll}
|x\rangle|b \oplus(F . x)\rangle & \text { if }\|x\| \leq k \\
|x\rangle|b\rangle & \text { if }\|x\|>k
\end{array} .\right.
$$

This operator requires at most $k$ queries, because the query complexity of $(F . x)$ is limited by the Hamming weight $\|x\|$ of $|x\rangle$, and can be carried out on states in superposition. The algorithm proceeds as follows:

- Prepare starting state $\left|\Psi_{k}\right\rangle \frac{1}{\sqrt{2}}(|0\rangle-|1\rangle)\left(\left|\Psi_{k}\right\rangle\right.$ depends on $k$, as is explained below)

- Act on state with $A_{k}$

- Apply $H^{\otimes N}$ to the first $N$ quibits

- Discard the ancilla qubit and measure the $N$ bits

For comparison with the previous two algorithms, we use the 'approximate oracle interrogation' version of WVD [3], for which the starting state $\Psi_{k}$ is prepared as follows:

$$
\Psi_{k}=\sum_{j=0}^{k} \frac{\alpha_{j}}{\sqrt{\left(\begin{array}{c}
N \\
m
\end{array}\right)}} \sum_{\substack{x \in\{0,1\}^{n} \\
\|x\|=j}}|x\rangle .
$$

Upon measurement, the observed state $\left|F^{\prime}\right\rangle$ is 'close' to $|F\rangle$, which we mean that we obtain an N-bit string of which $m$ bits are correct (that is, it shares $m$ bits with $F$, so correctly represents the function $f$ for $m$ values of $f(x))$ and the remainder incorrect, where we don't know in which locations the correct bits are. The probability distribution for $m$ is given by

$$
P(m)=\frac{\left(\begin{array}{c}
N \\
m
\end{array}\right)}{2^{N}}\left|\sum_{j=0}^{k} \frac{\alpha_{j}}{\sqrt{\left(\begin{array}{c}
k \\
m
\end{array}\right)}} \mathcal{K}_{j}(m ; N)\right|^{2}
$$

where $\mathcal{K}_{j}(m ; N)$ is a Krawtchouk polynomial [8] given by

$$
\mathcal{K}_{j}(m ; N)=\sum_{r=0}^{j}(-1)^{r}\left(\begin{array}{c}
m \\
r
\end{array}\right)\left(\begin{array}{c}
N-m \\
j-r
\end{array}\right) .
$$


We follow van Dam and use,

$$
\begin{array}{cl}
\alpha_{j}=\frac{1}{\sqrt{k}}, & k-\sqrt{k}+1 \leq j \leq k \\
0, & \text { otherwise. }
\end{array}
$$

This choice gives close to but not exactly the maximal expected number of correct bits; for example

$$
\begin{aligned}
\alpha_{j}=\sqrt{\frac{2}{k}}, & \frac{k}{2}+1 \leq j \leq k \\
0, & \text { otherwise }
\end{aligned}
$$

gives a higher expectation value. As an upper limit to the problem, for large $N$, the optimal expected number of correct bits is,

$$
E(m)_{o p t}=\frac{N}{2}+\sqrt{k} \sqrt{N-k+1} .
$$

As $\mathrm{k}$ increases the expected number of bits increases as shown in figure 5 .

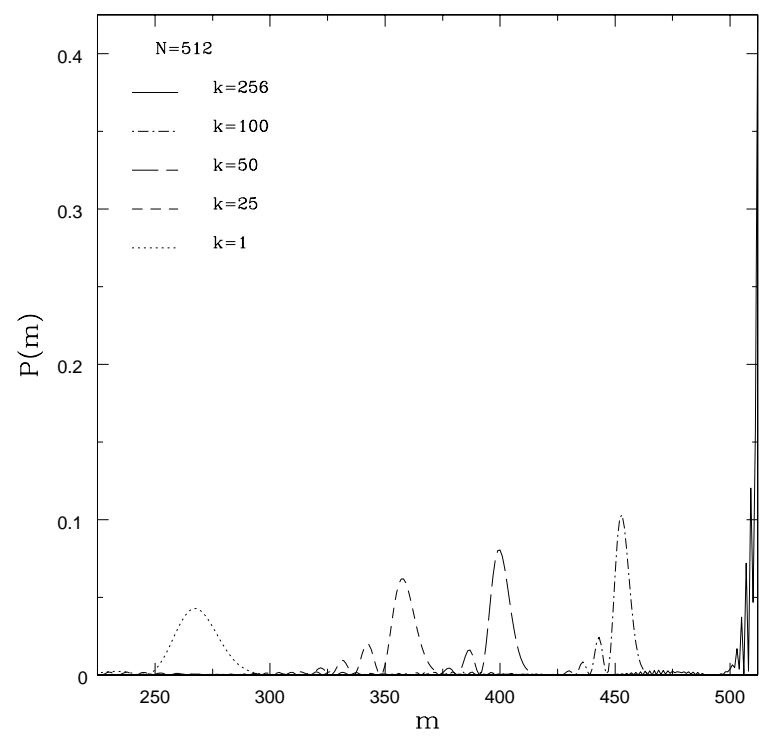

Figure 5: Probability distribution of the number of correct bits, m, known after k queries using the WVD algorithm for a string containing $\mathrm{N}=512$ bits.

Let us consider the function $f$ which is nearly balanced or constant with $N_{0}$ zeros. We tackle the DJ problem in a similar way to the classical algorithm as discussed in section 3.2 using an estimator for the proportion of 
zeros in the full string $\left(p_{0}=N_{0} / N\right)$. Our conclusions after $\mathrm{k}$ queries are based upon,

$$
\begin{aligned}
0.25 \leq \hat{p}_{0} \leq 0.75 & \text { infer balanced } \\
\hat{p}_{0}<0.25, \hat{p}_{0}>0.75 & \text { infer constant. }
\end{aligned}
$$

In the WVD algorithm the analogous estimator is $\hat{p}_{0}=m_{0} / N$, where $m_{0}$ is the number of zeros in the string after $\mathrm{k}$ queries. After $\mathrm{k}$ queries one obtains $m$ correct bits of which $m_{0}^{*}$ are correct zeros. We assume that the correct bits are drawn at random from the full population so that the distribution of correct zeros is given by,

$$
P\left(m_{0}^{*} \mid m\right)=\frac{\left(\begin{array}{c}
N_{0} \\
m_{0}^{*}
\end{array}\right)\left(\begin{array}{c}
N-N_{0} \\
m-m_{0}^{*}
\end{array}\right)}{\left(\begin{array}{l}
N \\
m
\end{array}\right)}
$$

with

$$
E\left(m_{0}^{*} \mid m\right)=\frac{N_{0}}{N} m
$$

In the resulting string from the WVD algorithm one can't distinguish correct from incorrect zeros; one purely measures the total number of zeros given by

$$
m_{0}=N-N_{0}-m+2 m_{0}^{*}
$$

where $m_{0}$ is distributed

$$
P\left(\hat{p}_{0}=\frac{m_{0}}{N}\right)=p\left(m_{0}\right)=\sum_{\text {all } m, m_{0}^{*}}^{\text {giving } m_{0}} P\left(m_{0}^{*} \mid m\right) P(m)
$$

with an expectation value,

$$
E\left(m_{0}\right)=N-N_{0}+2 E\left(m_{0}^{*}\right)-E(m)
$$

and an optimal value for the estimator of

$$
\hat{p}_{0, o p t}=E\left(\frac{m_{0}}{N}\right)_{o p t}=\frac{1}{2}+\left[\frac{2 N_{0}}{N}-1\right] \sqrt{\frac{k}{N}} \sqrt{1-\frac{k}{N}} .
$$

Figure 6] demonstrates that, in general, $m_{0}$ is not an unbiased estimator for $p_{0}$, in contrast to the classical estimator in section 3.2. Only as $\mathrm{k}$ increases does $m_{0}$ tend towards an unbiased estimator of $N_{0}$. Note that for a small number of queries this quantum estimator is much weaker than the classical, unbiased one in the cases where $N_{0}$ is significantly different from $N / 2$, i.e. for nearly constant strings. It is generally better in determining a near balanced string, however, where the $E\left(m_{0}\right)$ is close to $N_{0}$ even for small 

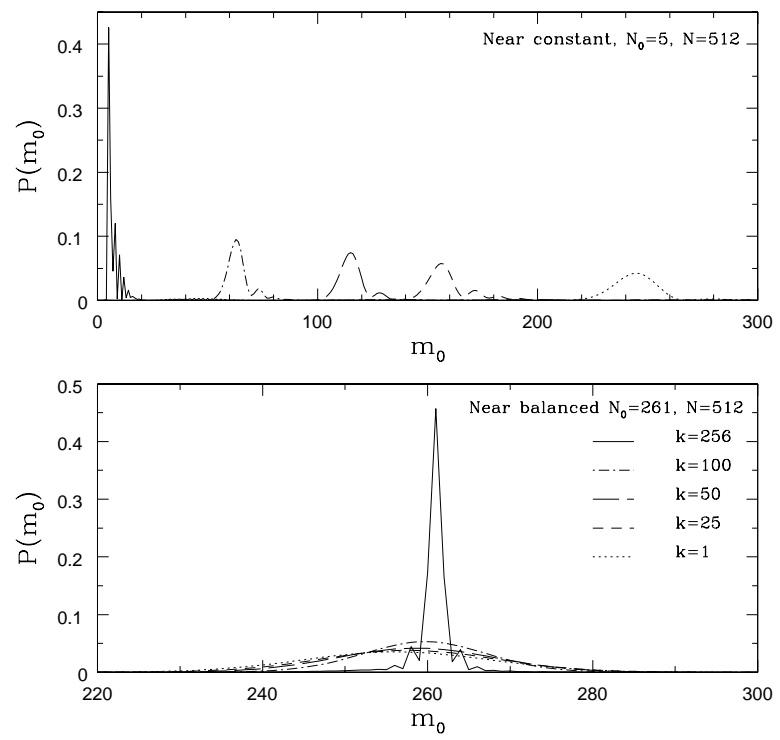

Figure 6: Probability distribution of the number of zeros in the string after $\mathrm{k}$ queries using the WVD algorithm for a string containing $\mathrm{N}=512$ bits for a nearly balanced string (lower panel) and a nearly constant string (upper panel).

query numbers, because its probability distribution has a smaller variance than the classical one.

So using equation (31), one considers the following failure probability when applying the WVD algorithm to the DJ problem with weakened promise:

$$
\begin{aligned}
P_{\text {fail }}= & \frac{1}{2}\left\{P\left(0.25 \leq \hat{p}_{0} \leq 0.75 \mid p_{0}<0.25, p_{0}>0.75\right)\right. \\
& \left.+P\left(\hat{p}_{0}<0.25, \hat{p}_{0}>0.75 \mid 0.25 \leq p_{0} \leq 0.75\right)\right\}
\end{aligned}
$$

where those quantities can be calculated by fixing $k$ in equation 19. $N_{0}$ in equation 30 and then using equation 31 as stated. The dominant error comes in correctly characterising a constant string, the first term in equation (34). In figures 7 and 8 we show the algorithm's behaviour as one varies the query number and degree of weakening. In figure 9 the algorithm is compared directly to the classical and DJ algorithms for two query numbers. One can see that the WVD algorithm never achieves the success rate of the DJ algorithm itself apart from at high $k$ and $y$ and never becomes superior to the classical algorithm. This is because we cannot tell which bits are correct in the in the $N$-bit string returned by the algorithm; if we did know, the algorithm would be better than both the classical and DJ algorithms. 


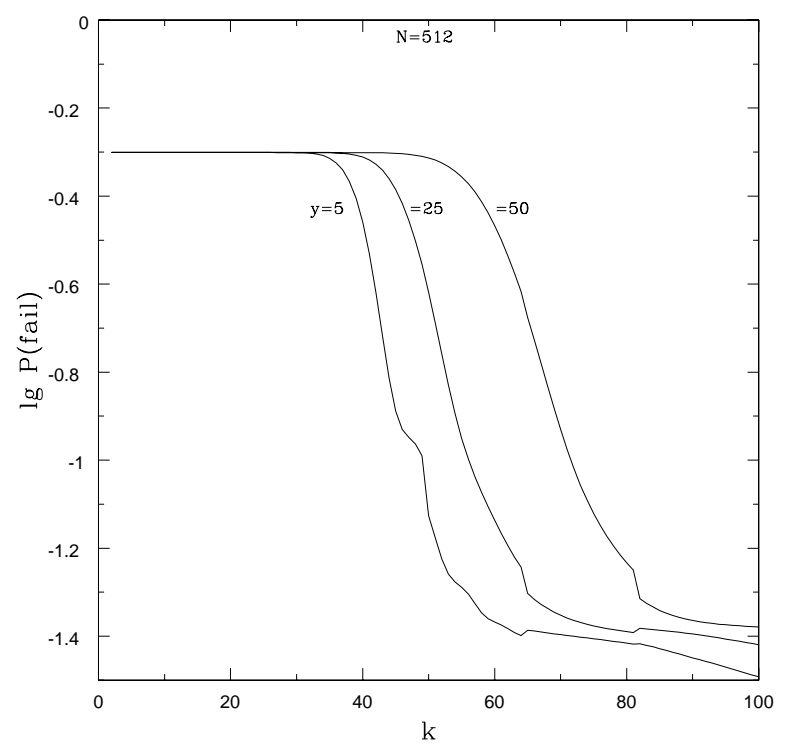

Figure 7: Failure probability for the WVD algorithm when applied to the DJ problem with weakened promise, as one varies the query number, $k$.

\section{Conclusion}

Figures [9 in which the relative performances of the three algorithms considered here are compared, illustrates that the problem is still structured; the DJ algorithm is successful in solving the problem but loses power as the promise becomes more broken. The WVD algorithm, designed for determining functions that have no promise on their nature, fails to produce good results except for in the limit of large number of queries, so that simply sampling bits classically and inferring from those is superior for the number of queries considered. Whilst the full version of the WVD algorithm would solve the problem well, that would require a number of queries over half that of the number of bits to sample, in which case classical sampling is very effective at solving the problem anyway.

We have found that the DJ algorithm is surprisingly robust when we consider breaks in the promise, with the failure probabilities driven predominantly by a reduction in correctly inferring a constant function. The speed up in comparison to a classical sampling algorithm is obviously reduced but one still retains an advantage for smaller weakenings in the promise.

When compared against the quantum algorithm tailored to unstructured problems, WVD, both the classical and DJ algorithms outperform it for small query numbers and low degree of weakening in the promise. The main downfall of the WVD algorithm in solving this problem is that with a small 


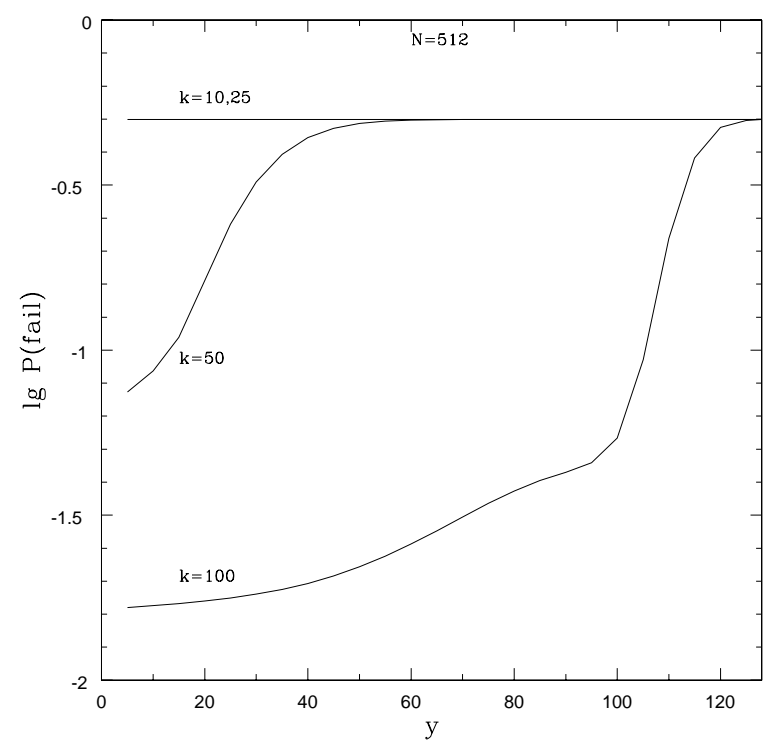

Figure 8: Failure probability for the WVD algorithm when applied to the DJ problem with weakened promise, as one varies the degree of weakening, $y$.

query number there still remains a fairly large proportion of incorrectly known bits which severely distorts the sample distribution. The nature of the problem does not lend itself to simply producing a function description that contains errors, as the WVD algorithm does, although perhaps the underlying principles of the WVD algorithm would allow modification in the algorithm to achieve greater efficacy. The DJ algorithm, designed for the unbroken promise, nevertheless retains much of its advantage in the case where the promise is not greatly weakened.

\section{Ackowledgements}

We thank Peter L Knight for useful discussions and Wim van Dam for communications including sharing details of his calculations on the success probability of his algorithm from his $\mathrm{PhD}$ thesis. AB acknowledges financial support from the Engineering and Physical Sciences Research Council (EPSRC). 


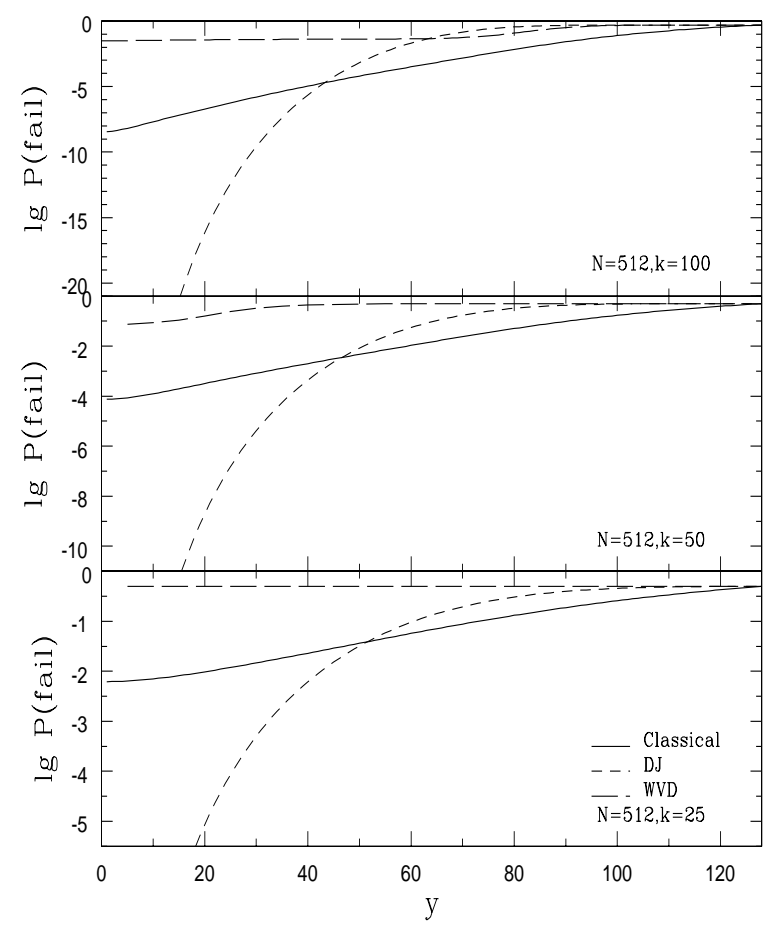

Figure 9: Comparison of failure probabilities for the classical, DJ and WVD quantum algorithms with $N=512$, and $k=25$ (lower panel), $k=50$ (middle panel) or $k=100$ (upper panel).

\section{References}

[1] R. Beals, H. Buhrman, R. Cleve, M. Mosca and R. De Wolf Quantum Lower Bounds by Polynomials quant-ph/9802049

[2] J. Preskill Lecture Notes on Quantum Computation, Physics 219 (Available at www.theory.caltech.edu/people/preskill/ph229/\#lecture, 1997)

[3] Wim van Dam Quantum Oracle Interrogation: Getting all the information for almost half the price. Proceedings of FOCS'98, 8-11 Novemember in Palo Alto, CA

[4] D. Deutsch Quantum Theory, the Church-Turing Principle and the universal quantum computer Proc. R. Soc. Lond. A, 400:97, 1985

[5] D.Deutsch and R. Jozsa Rapid solutions of problems by quantum parallelism Proc. R. Soc. Lond. A, 439:553, 1992 
[6] R.Cleve, A.Ekert, C.Machiavellio and M.Mosca Quantum algorithms revisited Proc. R. Soc. Lond. A, 454(1969):339-354, 1998

[7] Wim van Dam, Appendix D in On Quantum Computation Theory, Ph.D. thesis, ILLC Dissertation Series 2002-04, University of Amsterdam, The Netherlands, 2002

[8] I. Gradshteyn and I. Ryzhik Table of Integrals, Series, and Products Academic Press, 1965 